\title{
Noncommutative integrability on noncompact invariant manifolds
}

\author{
E.Fiorani ${ }^{1}$, G.Sardanashvily ${ }^{2}$ \\ ${ }^{1}$ Department of Mathematics and Informatics, University of Camerino, 62032 Camerino (MC), \\ Italy \\ ${ }^{2}$ Department of Theoretical Physics, Moscow State University, 117234 Moscow, Russia
}

\begin{abstract}
The Mishchenko-Fomenko theorem on noncommutative completely integrable Hamiltonian systems on a symplectic manifold is extended to the case of noncompact invariant submanifolds.
\end{abstract}

PACS numbers: 45.20.Jj, 02.30.Ik

\section{Introduction}

We are concerned with the classical theorems on abelian and noncommutative integrability of Hamiltonian systems on a symplectic manifold. These are the Liouville-Arnold theorem on abelian completely integrable systems (henceforth CIS) [1, 2, 26], the PoincaréLyapounov-Nekhoroshev theorem on abelian partially integrable systems [17, 31, 32], and the Mishchenko-Fomenko one on nocommutative CISs [14, 25, 30]. These theorems state the existence of (generalized) action-angle coordinates around a compact invariant submanifold, which is a torus. However, there is a topological obstruction to the existence of global action-angle coordinates [10, 11]. The Liouville-Arnold and Nekhoroshev theorems have been extended to noncompact invariant submanifolds, which are toroidal cylinders $[15,16,22,38]$. In particular, this is the case of time-dependent CISs [21, 23]. Any timedependent CIS of $m$ degrees of freedom can be represented as an autonomous one of $m+1$ degrees of freedom on a homogeneous momentum phase space, where time is a generalized angle coordinate. Therefore, we further consider autonomous CISs.

Our goal here is the following generalization of the Mishchenko-Fomenko theorem to noncommutative CISs whose invariant submanifolds need not be compact.

Theorem 1. Let $(Z, \Omega)$ be a connected symplectic $2 n$-dimensional real smooth manifold and $\left(C^{\infty}(Z),\{\},\right)$ the Poisson algebra of smooth real functions on $Z$. Let a subset $H=$ $\left(H_{1}, \ldots, H_{k}\right), n \leq k<2 n$, of $C^{\infty}(Z)$ obey the following conditions. 
(i) The Hamiltonian vector fields $\vartheta_{i}$ of functions $H_{i}$ are complete.

(ii) The map $H: Z \rightarrow \mathbb{R}^{k}$ is a submersion with connected and mutually diffeomorphic fibers, i.e.,

$$
H: Z \rightarrow N=H(Z)
$$

is a fibered manifold over a connected open subset $N \subset \mathbb{R}^{k}$.

(iii) There exist real smooth functions $s_{i j}: N \rightarrow \mathbb{R}$ such that

$$
\left\{H_{i}, H_{j}\right\}=s_{i j} \circ H, \quad i, j=1, \ldots, k .
$$

(iv) The matrix function with the entries $s_{i j}$ (2) is of constant corank $m=2 n-k$ at all points of $N$.

Then the following hold.

(I) The fibers of $H$ (1) are diffeomorphic to a toroidal cylinder

$$
\mathbb{R}^{m-r} \times T^{r}
$$

(II) Given a fiber $M$ of $H$ (1), there exists an open saturated neighbourhood $U_{M}$ of it (i.e., a fiber through a point of $U_{M}$ belongs to $U_{M}$ ), which is a trivial principal bundle with the structure group (3).

(III) Given standard coordinates $\left(y^{\lambda}\right)$ on the toroidal cylinder (3), the neighbourhood $U_{M}$ is provided with bundle coordinates $\left(J_{\lambda}, p_{A}, q^{A}, y^{\lambda}\right)$, called the generalized action-angle coordinates, which are the Darboux coordinates of the symplectic form $\Omega$ on $U_{M}$, i.e.,

$$
\Omega=d J_{\lambda} \wedge d y^{\lambda}+d p_{A} \wedge d q^{A}
$$

In Hamiltonian mechanics, one can think of functions $H_{i}$ in Theorem 1 as being integrals of motion of a CIS. Their level surfaces (fibers of $H$ ) are invariant submanifolds of a CIS.

\section{Abelian completely and partially integrable systems}

The proof of Theorem 1 is based on the fact that an invariant submanifold of a noncommutative CIS is a maximal integral manifold of some abelian partially integrable Hamiltonian system [14].

If $k=n$, Theorem 1 provides the above mentioned extension of the Liouville-Arnold theorem to abelian CISs whose invariant submanifolds are noncompact ([38], Theorem 6.1; [15], Theorem 1). Note that the proof of Theorem 6.1 [38] differs from that of Theorem 
1 [15]. It is based on Lemma 6.4. The statement of its Corollary 6.3 is equivalent to the assumption of Lemmas $6.1-6.4$ that an imbedded invariant submanifold $N_{x} \subset K$ admits a Lagrangian transversal submanifold $W \subset K$ through $x$. Apparently, one can avoid the construction of $T^{*}(W)$ from the proof and, instead of the map $\gamma$, consider the map

$$
W \times \mathbb{R}^{n} \longrightarrow\left(W \times \mathbb{R}^{n}\right) / \mathbb{Z}^{m(x)} \longrightarrow \alpha\left(W \times \mathbb{R}^{n}\right)
$$

One also need not appeal to the concept of many-valued functions $\varphi_{i}$, but can show that the fibered manifold $\alpha\left(W \times \mathbb{R}^{n}\right) \rightarrow W$ is a fiber bundle. This is always true if its fibers are tori and, if $m(x)<n$, follows from the fact that sections $l_{i}$ of $W \times \mathbb{R}^{n} \rightarrow W$ introduced in Lemma 6.2 are smooth.

The condition (ii) of Theorem 1 implies that the functions $\left\{H_{\lambda}\right\}$ are independent on $Z$,

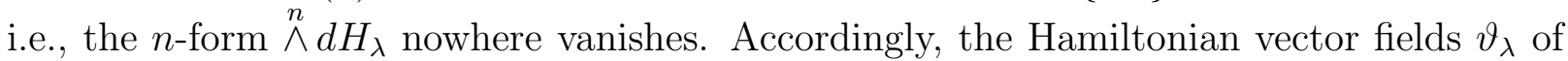
these functions are independent on $Z$, i.e., the multivector field $\wedge^{n} \vartheta_{\lambda}$ nowhere vanishes. If $k=n$, these vector fields are mutually commutative, and they span a regular involutive $n$-dimensional distribution on $Z$ whose maximal integral manifolds are exactly fibers of the fibered manifold (1). Thus, every fiber of $H$ (1) admits $n$ independent complete vector fields, i.e., it is a locally affine manifold and, consequently, diffeomorphic to a toroidal cylinder.

Considering an abelian CIS around some compact invariant submanifold, we come to the Liouville-Arnold theorem (somebody also calls it the Liouville-Mineur-Arnold theorem [39]). Instead of the conditions (i) and (ii) of Theorem 1, one can suppose that integrals of motion $\left\{H_{\lambda}\right\}$ are independent almost everywhere on a symplectic manifold $Z$, i.e., the set of points where the exterior form $\stackrel{n}{\wedge} d H_{\lambda}$ (or, equivalently, the multivector field $\stackrel{n}{\wedge} \vartheta_{\lambda}$ ) vanishes is nowhere dense. In this case, connected components of level surfaces of functions $\left\{H_{\lambda}\right\}$ form a singular Stefan foliation $\mathcal{F}$ of $Z$ whose leaves are both the maximal integral manifolds of the singular involutive distribution spanned by the vector fields $\vartheta_{\lambda}$ and the orbits of the pseudogroup $G$ of local diffeomorphisms of $Z$ generated by flows of these vector fields [36, 37]. Let $M$ be a leaf of $\mathcal{F}$ through a regular point $z \in Z$ where $\stackrel{n}{\wedge} \vartheta_{\lambda} \neq 0$. It is regular everywhere because the group $G$ preserves $\stackrel{n}{\wedge} \vartheta_{\lambda}$. If $M$ is compact and connected, there exists its saturated open neighbourhood $U_{M}$ such that the map $H$ restricted to $U_{M}$ satisfies the condition (ii) of Theorem 1, i.e., the foliation $\mathcal{F}$ of $U_{M}$ is a fibered manifold in tori $T^{n}$. Since its fibers are compact, $U_{M}$ is a bundle [29]. Hence, it contains a saturated open neighbourhood of $M$, say again $U_{M}$, which is a trivial principal bundle with the structure group $T^{n}$. Providing $U_{M}$ with the Darboux (action-angle) coordinates $\left(J_{\lambda}, \alpha^{\lambda}\right)$, one uses the fact that there are no linear functions on a torus $T^{n}$. 
The Poincaré-Lyapounov-Nekhoroshev theorem generalizes the Liouville-Arnold one to partially integrable systems characterized by $k<n$ independent integrals of motion $H_{\lambda}$ in involution. In this case, one deals with $k$-dimensional maximal integral manifolds of the distribution spanned by Hamiltonian vector fields $\vartheta_{\lambda}$ of integrals of motion $H_{\lambda}$. The Poincaré-Lyapounov-Nekhoroshev theorem imposes a sufficient condition which Hamiltonian vector fields $\vartheta_{\lambda}$ must satisfy in order that their compact maximal integral manifold $M$ admits an open neighbourhood fibered in tori $[17,18]$. Such a condition has been also investigated in the case of noncommutative vector fields depending on parameters [19]. Extending the Poincaré-Lyapounov-Nekhoroshev theorem to the case of noncompact integral submanifolds, we in fact assumed from the beginning that these submanifolds form a fibration $[15,22,23]$. In a more general setting, we have studied the property of a given dynamical system to be Hamiltonian relative to different Poisson structures [5, 22, 35]. As is well known, any integrable Hamiltonian system is Hamiltonian relative to different symplectic and Poisson structures, whose variety has been analyzed from different viewpoints $[3,4,8,12,13,28,34]$. One of the reasons is that bi-Hamiltonian systems have a large supply of integrals of motion. Here, we refer to our following result on partially integrable systems on a symplectic manifold ([22], Theorem 6).

Theorem 2. Given a 2n-dimensional symplectic manifold $(Z, \Omega)$, let $\left\{H_{1}, \ldots, H_{m}\right\}$, $m \leq n$, be smooth real functions on $Z$ in involution which satisfy the following conditions.

(i) The functions $H_{\lambda}$ are everywhere independent.

(ii) Their Hamiltonian vector fields $\vartheta_{\lambda}$ are complete.

(iii) These vector fields span a regular distribution whose maximal integral manifolds form a fibration $\mathcal{F}$ of $Z$ with diffeomorphic fibers.

Then the following hold.

(I) All fibers of $\mathcal{F}$ are diffeomorphic to a toroidal cylinder (3).

(II) There is an open saturated neighbourhood $U_{M}$ of every fiber $M$ of $\mathcal{F}$ which is a trivial principal bundle with the structure group (3).

(III) Given standard coordinates $\left(y^{\lambda}\right)$ on the toroidal cylinder (3), the neighbourhood $U_{M}$ is endowed with the bundle coordinates $\left(J_{\lambda}, p_{A}, q^{A}, y^{\lambda}\right)$ such that the symplectic form $\Omega$ is brought into the form (4).

Theorem 2 provides the above mentioned generalization of the Poincaré-LyapounovNekhoroshev theorem to the case of noncompact invariant submanifolds. A geometric aspect of this generalization is the following. Any fibered manifold whose fibers are diffeomorphic either to $\mathbb{R}^{r}$ or a compact connected manifold $K$ (e.g., a torus) is a fiber bundle 
[29]. However, a fibered manifold whose fibers are diffeomorphic to a product $\mathbb{R}^{r} \times K$ (e.g., a toroidal cylinder (3)) need not be a fiber bundle (see [20], Example 1.2.2).

\section{The proof of Theorem 1}

Theorem 2 is the final step of the proof of Theorem 1. The condition (iv) of Theorem 1 implies that an $m$-dimensional invariant submanifold of a noncommutative CIS is a maximal integral manifold of some abelian partially integrable Hamiltonian system obeying the conditions of Theorem 2. The proof of this fact is based on the following two assertions $[14,27]$.

Lemma 3. Given a symplectic manifold $(Z, \Omega)$, let $H: Z \rightarrow N$ be a fibered manifold such that, for any two functions $f, f^{\prime}$ constant on fibers of $H$, their Poisson bracket $\left\{f, f^{\prime}\right\}$ is so. Then $N$ is provided with an unique coinduced Poisson structure $\{,\}_{N}$ such that $H$ is a Poisson morphism.

Since any function constant on fibers of $H$ is a pull-back of some function on $N$, the condition of Lemma 3 is satisfied due to item (iii) of Theorem 1. Thus, the base $N$ of the fibration (1) is endowed with a coinduced Poisson structure.

Lemma 4. Given a fibered manifold $H: Z \rightarrow N$, the following conditions are equivalent:

(i) the rank of the coinduced Poisson structure $\{,\}_{N}$ on $N$ equals $2 \operatorname{dim} N-\operatorname{dim} Z$,

(ii) the fibers of $H$ are isotropic,

(iii) the fibers of $H$ are maximal integral manifolds of the involutive distribution spanned by the Hamiltonian vector fields of the pull-back $H^{*} C$ of Casimir functions $C$ of the Poisson algebra on $N$.

It is readily observed that the condition (i) of Lemma 4 is satisfied due to the assumption (iv) of Theorem 1. It follows that every fiber $M$ of the fibration (1) is a maximal integral manifold of the involutive distribution spanned by the Hamiltonian vector fields $v_{\lambda}$ of the pull-back $H^{*} C_{\lambda}$ of $m$ independent Casimir functions $\left\{C_{1}, \ldots, C_{m}\right\}$ on an open neighbourhood $N_{M}$ of the point $H(M)$. Let us put $U_{M}=H^{-1}\left(N_{M}\right)$. It is an open saturated neighbourhood of $M$. Since

$$
H^{*} C_{\lambda}(z)=\left(C_{\lambda} \circ H\right)(z)=C_{\lambda}\left(H_{i}(z)\right), \quad z \in U_{M}
$$

the Hamiltonian vector fields $v_{\lambda}$ on $M$ are linear combinations of Hamiltonian vector fields $\vartheta_{i}$ of the functions $H_{i}$ and, therefore, they are complete on $M$. Similarly, they are complete 
on any fiber of $U_{M}$ and, consequently, on $U_{M}$. Thus, the conditions of Theorem 2 hold on $U_{M}$. This completes the proof of Theorem 1 .

The proof of Theorem 1 gives something more. Let $\left\{H_{i}\right\}$ be integrals of motion of a Hamiltonian $\mathcal{H}$. Since $\left(J_{\lambda}, p_{A}, q^{A}\right)$ are coordinates on $N$, they are also integrals of motion of $\mathcal{H}$. Therefore, the Hamiltonian $\mathcal{H}$ depends only on the action coordinates $J_{\lambda}$, and the equation of motion read

$$
\dot{y}^{\lambda}=\frac{\partial \mathcal{H}}{\partial J_{\lambda}}, \quad J_{\lambda}=\text { const. }, \quad q^{A}=\text { const. }, \quad p_{A}=\text { const. }
$$

Though the integrals of motion $H_{i}$ are smooth functions of coordinates $\left(J_{\lambda}, q^{A}, p_{A}\right)$, the Casimir functions

$$
C_{\lambda}\left(H_{i}\left(J_{\mu}, q^{A}, p_{A}\right)\right)=C_{\lambda}\left(J_{\mu}\right)
$$

depend only on the action coordinates $J_{\lambda}$. Moreover, a Hamiltonian

$$
\mathcal{H}\left(J_{\mu}\right)=\mathcal{H}\left(C_{\lambda}\left(H_{i}\left(J_{\mu}, q^{A}, p_{A}\right)\right)\right.
$$

is expressed in integrals of motion $H_{i}$ through the Casimir functions (5).

Let us note that, under the assumptions of the Mishchenko-Fomenko theorem, a noncommutative CIS is also integrable in the abelian sense. Namely, it admits $n$ independent integrals of motion in involution [6]. Under the conditions of Theorem 1, such integrals of motion in involution exist, too. All of them are the pull-back of functions on $N$. However, one must justify that they obey the condition (iii) of Theorem 2 in order to characterize an abelian CIS.

\section{Example}

The original Mishchenko-Fomenko theorem is restricted to CISs whose integrals of motion $\left\{H_{1}, \ldots, H_{k}\right\}$ form a $k$-dimensional real Lie algebra $\mathcal{G}$ of rank $m$ with the commutation relations

$$
\left\{H_{i}, H_{j}\right\}=c_{i j}^{h} H_{h}, \quad c_{i j}^{h}=\text { const. }
$$

In this case, nonvanishing complete Hamiltonian vector fields $\vartheta_{i}$ of $H_{i}$ define a free Hamiltonian action on $Z$ of some connected Lie group $G$ whose Lie algebra is isomorphic to $\mathcal{G}$. Orbits of $G$ coincide with $k$-dimensional maximal integral manifolds of the regular distribution on $Z$ spanned by Hamiltonian vector fields $\vartheta_{i}$ [37]. Furthermore, one can treat $H$ 
(1) as an equivariant momentum mapping of $Z$ to the Lie coalgebra $\mathcal{G}^{*}$, provided with the coordinates $x_{i}(H(z))=H_{i}(z), z \in Z,[23,24]$. In this case, the coinduced Poisson structure $\{,\}_{N}$ in Lemma 3 coincides with the canonical Lie-Poisson structure on $\mathcal{G}^{*}$ given by the Poisson bivector field

$$
w=\frac{1}{2} c_{i j}^{h} x_{h} \partial^{i} \wedge \partial^{j} .
$$

Recall that the coadjoint action of $\mathcal{G}$ on $\mathcal{G}^{*}$ reads $\varepsilon_{i}\left(x_{j}\right)=c_{i j}^{h} x_{h}$, where $\left\{\varepsilon_{i}\right\}$ is a basis for $\mathcal{G}$. Casimir functions of the Lie-Poisson structure are exactly the coadjoint invariant functions on $\mathcal{G}^{*}$. They are constant on orbits of the coadjoint action of $G$ on $\mathcal{G}^{*}$ which coincide with leaves of the symplectic foliation of $\mathcal{G}^{*}$. Given a point $z \in Z$ and the orbit $G_{z}$ of $G$ in $Z$ through $z$, the fibration $H$ (1) projects this orbit onto the orbit $G_{H(z)}$ of the coadjoint action of $G$ in $\mathcal{G}^{*}$ through $H(z)$. Moreover, by virtue of item (iii), Lemma 4, the inverse image $H^{-1}\left(G_{H(z)}\right)$ of $G_{H(z)}$ coincides with the orbit $G_{z}$. It follows that any orbit of $G$ in $Z$ is fibered in invariant submanifolds.

The Mishchenko-Fomenko theorem has been mainly applied to CISs whose integrals of motion form a compact Lie algebra. Indeed, the group $G$ generated by flows of the Hamiltonian vector fields is compact, and every orbit of $G$ in $Z$ is compact. Since a fibration of a compact manifold possesses compact fibers, any invariant submanifold of such a noncommutative CIS is compact. Therefore, our Theorem 1 essentially extends a class of noncommutative CISs under investigation.

For instance, a spherical top exemplifies a noncommutative CIS whose integrals of motion make up the compact Lie algebra so(3) with respect to some symplectic structure.

Let us consider a CIS with the Lie algebra $\mathcal{G}=s o(2,1)$ of integrals of motion $\left\{H_{1}, H_{2}, H_{3}\right\}$ on a four-dimensional symplectic manifold $(Z, \Omega)$, namely,

$$
\left\{H_{1}, H_{2}\right\}=-H_{3}, \quad\left\{H_{2}, H_{3}\right\}=H_{1}, \quad\left\{H_{3}, H_{1}\right\}=H_{2} .
$$

The rank of this algebra (the dimension of its Cartan subalgebra) equals one. Therefore, an invariant submanifold in Theorem 1 is $M=\mathbb{R}$, provided with a Cartesian coordinate $y$. Let us consider its open saturated neighbourhood $U_{M}$ projected via $H: U_{M} \rightarrow N$ onto a domain $N \subset \mathcal{G}^{*}$ in the Lie coalgebra $\mathcal{G}^{*}$ centered at a point $H(M) \in \mathcal{G}^{*}$ which belongs to an orbit of the coadjoint action of maximal dimension 2. A domain $N$ is endowed with the coordinates $\left(x_{1}, x_{2}, x_{3}\right)$ such that integrals of motion $\left\{H_{1}, H_{2}, H_{3}\right\}$ on $U_{M}=N \times \mathbb{R}$, coordinated by $\left(x_{1}, x_{2}, x_{3}, y\right)$, read

$$
H_{1}=x_{1}, \quad H_{2}=x_{2}, \quad H_{3}=x_{3} .
$$


As was mentioned above, the coinduced Poisson structure on $N$ is the Lie-Poisson structure

$$
w=x_{2} \partial^{3} \wedge \partial^{1}-x_{3} \partial^{1} \wedge \partial^{2}+x_{1} \partial^{2} \wedge \partial^{3} .
$$

Let us endow $N$ with different coordinates $\left(r, x_{1}, \gamma\right)$ given by the equalities

$$
r=\left(x_{1}^{2}+x_{2}^{2}-x_{3}^{2}\right)^{1 / 2}, \quad x_{2}=\left(r^{2}-x_{1}^{2}\right)^{1 / 2} \operatorname{ch} \gamma, \quad x_{3}=\left(r^{2}-x_{1}^{2}\right)^{1 / 2} \operatorname{sh} \gamma,
$$

where $r$ is a Casimir function on $\mathcal{G}^{*}$. It is readily observed that the coordinates $(8)$ are the Darboux coordinates of the Lie-Poisson structure (7), namely,

$$
w=\frac{\partial}{\partial \gamma} \wedge \frac{\partial}{\partial x_{1}}
$$

Let $\vartheta_{r}$ be the Hamiltonian vector field of the Casimir function $r$ (8). This vector field is a combination

$$
\vartheta_{r}=\frac{1}{r}\left(x_{1} \vartheta_{1}+x_{2} \vartheta_{2}-x_{3} \vartheta_{3}\right)
$$

of the Hamiltonian vector fields $\vartheta_{i}$ of integrals of motion $H_{i}$. Its flows are invariant submanifolds. Let $y$ be a parameter along the flows of this vector field, i.e.,

$$
\vartheta_{r}=\frac{\partial}{\partial y}
$$

Then the Poisson bivector associated to the symplectic form $\Omega$ on $U_{M}$ is

$$
W=\frac{\partial}{\partial r} \wedge \frac{\partial}{\partial y}+\frac{\partial}{\partial \gamma} \wedge \frac{\partial}{\partial x_{1}}
$$

Accordingly, Hamiltonian vector fields of integrals of motion take the form

$$
\begin{aligned}
& \vartheta_{1}=-\frac{\partial}{\partial \gamma} \\
& \vartheta_{2}=r\left(r^{2}-x_{1}^{2}\right)^{-1 / 2} \operatorname{ch} \gamma \frac{\partial}{\partial y}+x_{1}\left(r^{2}-x_{1}^{2}\right)^{-1 / 2} \operatorname{ch} \gamma \frac{\partial}{\partial \gamma}+\left(r^{2}-x_{1}^{2}\right)^{1 / 2} \operatorname{sh} \gamma \frac{\partial}{\partial x_{1}}, \\
& \vartheta_{3}=r\left(r^{2}-x_{1}^{2}\right)^{-1 / 2} \operatorname{sh} \gamma \frac{\partial}{\partial y}+x_{1}\left(r^{2}-x_{1}^{2}\right)^{-1 / 2} \operatorname{sh} \gamma \frac{\partial}{\partial \gamma}+\left(r^{2}-x_{1}^{2}\right)^{1 / 2} \operatorname{ch} \gamma \frac{\partial}{\partial x_{1}} .
\end{aligned}
$$

Thus, a symplectic annulus $\left(U_{M}, W\right)$ around an invariant submanifold $M=\mathbb{R}$ is endowed with the generalized action-angle coordinates $\left(r, x_{1}, \gamma, y\right)$, and possesses the corresponding noncommutative CIS $\left\{r, H_{1}, \gamma\right\}$ with the commutation relations

$$
\left\{r, H_{1}\right\}=0, \quad\{r, \gamma\}=0, \quad\left\{H_{1}, \gamma\right\}=1 .
$$

This CIS is related to the original one by the transformations

$$
r=\left(H_{1}^{2}+H_{2}^{2}-H_{3}^{2}\right)^{1 / 2}, \quad H_{2}=\left(r^{2}-H_{1}^{2}\right)^{1 / 2} \operatorname{ch} \gamma, \quad H_{3}=\left(r^{2}-H_{1}^{2}\right)^{1 / 2} \operatorname{ch} \gamma .
$$

Its Hamiltonian is expressed only in the action variable $r$. 


\section{References}

[1] Arnold V and Avez A 1968 Ergodic Problems in Classical Mechanics (Benjamin: NY)

[2] Arnold V (Ed.) 1990 Dynamical Systems III, IV (Springer: Berlin)

[3] Bogoyavlenskij O 1996 Theory of tensor invariants of integrable hamiltonian systems. I. Incompatible Poisson structures Commun. Math. Phys. 180 529-586

[4] Bogoyavlenskij O 1998 Extended integrability and bi-Hamiltonian systems Commun. Math. Phys. 196 19-51

[5] Bolsinov A and Borisov A 2002 Compatible Poisson brackets on Lie algebras Math. Notes 72 10-30

[6] Bolsinov A and Jovanović B 2003 Noncommutative integrability, moment map and geodesic flows Ann. Global Anal. Geom. 23 305-322

[7] Bolsinov A and Jovanović B 2004 Complete involutive algebras of functions on cotangent bundles of homogeneous spaces Math. Z 246 213-236

[8] Brouzet R 1993 About the existence of recursion operators for completely integrable Hamiltonian systems near a Liouville torus J. Math. Phys. 34 1309-1313

[9] Cushman R and Bates L 1997 Global Aspects of Classical Integrable Systems (Birkhäuser: Basel)

[10] Dazord P and Delzant T 1987 Le probleme general des variables actions-angles J. Diff. Geom. 26 223-251

[11] Dustermaat J 1980 On global action-angle coordinates Commun. Pure Appl. Math. 33 687-706

[12] Fassó F and Ratiu T 1998 Compatibility of symplectic structures adapted to noncommutatively integrable systems J. Geom. Phys. 27 199-220

[13] Fassó F and Giacobbe A 2002 Geometric structure of "broadly integrable" vector fields J. Geom. Phys. 44 156-170

[14] Fassó F 2005 Superintegrable Hamiltonian systems: geometry and applications Acta Appl. Math. 87 93-121 
[15] Fiorani E, Giachetta G and Sardanashvily G 2003 The Liouville-Arnold-Nekhoroshev theorem for non-compact invariant manifolds J. Phys. A 36 L101-L107

[16] Fiorani E 2004 Completely and partially integrable systems in the noncompact space Int. J. Geom. Methods Mod. Phys. 1 167-183

[17] Gaeta G 2002 The Poincaré-Lyapunov-Nekhoroshev theorem Ann. Phys. 297 157-173

[18] Gaeta G 2003 The Poincaré-Nekhoroshev map J. Nonlin. Math. Phys. 10 51-64

[19] Gaeta G 2006 The Poincaré-Lyapunov-Nekhoroshev theorem for involutory systems of vector fields Ann. Phys. 321 1277-1296

[20] Giachetta G, Mangiarotti L and Sardanashvily G 1997 New Lagrangian and Hamiltonian Methods in Field Theory (World Scientific: Singapore)

[21] Giachetta G, Mangiarotti L. and Sardanashvily G 2002 Action-angle coordinates for time-dependent completely integrable Hamiltonian systems, J. Phys. A 35 L439-L445

[22] Giachetta G, Mangiarotti L and Sardanashvily G 2003 Bi-Hamiltonian partially integrable systems J. Math. Phys. 44 1984-1997

[23] Giachetta G, Mangiarotti L and Sardanashvily G 2005 Geometric and Algebraic Topological Methods in Quantum Mechanics (World Scientific: Singapore)

[24] Guillemin V and Sternberg S 1984 Symplectic Techniques in Physics (Cambr. Univ. Press: Cambridge)

[25] Karasev M and Maslov V 1993 Nonlinear Poisson brackets. Geometry and quantization Translations of AMS 119 (AMS: Providence, RI)

[26] Lazutkin V 1993 KAM Theory and Semiclassical Approximations to Eigenfunctions (Springer: Berlin)

[27] Libermann P and Marle C-M 1987 Symplectic Geometry and Analytical Mechanics (D.Reidel Publishing Company: Dordrecht)

[28] Magri F 1978 A simple model of the integrable Hamiltonian equations J. Math. Phys. $19176-178$

[29] Meigniez G 2002 Submersion, fibration and bundles Trans. Amer. Math. Soc. 354 3771-3787 
[30] Mishchenko A and Fomenko A 1978 Generalized Liouville method of integration of Hamiltonian systems Funct. Anal. Appl. 12 113-121

[31] Nekhoroshev N 1972 Action-angle variables and their generalization Trans. Mos. Math. Soc. 26 180-198

[32] Nekhoroshev N 1994 The Poincaré-Lyapunov-Liouville-Arnol'd theorem Funct. Anal. Appl. 28 (1994) 128-129

[33] Sardanashvily G 1998 Hamiltonian time-dependent mechanics J. Math. Phys. 39 27142729

[34] Smirnov R 1996 On the master symmetries related to a certain classes of integrable hamiltonian systems J. Phys. A 29 8133-8138

[35] Sparano G and Vilasi G 2000 Noncommutative integrability and recursion operators J. Geom. Phys. 36 270-284

[36] Stefan A 1974 Accesible sets, orbits and foliations with singularities Proc. London Math. Soc. 29 699-713

[37] Sussmann H 1973 Orbits of families of vector fields and integrability of distributions Trans. Amer. Math. Soc. 180 171-188

[38] Vinogradov A and Kupershmidt B 1977 The structures of Hamiltonian mechanics Russian Math. Surveys 32 (4) 177-244

[39] Zung Nguyen Tien 2005 Torus actions and integrable systems In vol. Topological Methods in Theory of Integrable Systems Eds. A. Bolsinov, A.Fomenko and A.Oshemkov (Cambr. Sci. Publ.: Cambridge); Preprint math.DS/0407455 